\title{
Conocimiento y prácticas de prevención sobre el virus del papiloma humano (VPH) en universitarios de la Sierra Sur, Oaxaca
} Human papillomavirus (HPV) knowledge and prevention practices among Sierra Sur, Oaxaca college students

\author{
Gisela Mayra Bustamante-Ramos ${ }^{1}$, Abisai Martínez-Sánchez¹, Inés Tenahua-Quitl², \\ Concepción Jiménez ${ }^{1, a}$, Yarely López Mendoza ${ }^{1, a}$ \\ ${ }^{1}$ Instituto de Investigación sobre la Salud Pública (IISSP), Universidad de la Sierra Sur, Oaxaca, México. \\ ${ }^{2}$ Facultad de Enfermeria, Benemérita Universidad Autónoma de Puebla, México. \\ ${ }^{a}$ Estudiante de la licenciatura en Enfermería.
}

\begin{abstract}
Resumen
Introducción. El virus del papiloma humano (VPH) ocasiona la infección de transmisión sexual más importante en el mundo y se le considera como el principal agente para el desarrollo del cáncer cervicouterino. Los jóvenes constituyen la población más vulnerable debido al inicio de las relaciones sexuales usualmente sin métodos de protección. Objetivos. Describir el conocimiento para la prevención del virus del papiloma humano en universitarios de la sierra sur, Oaxaca. Diseño. Estudio cuantitativo, descriptivo de corte transversal. Lugar. Sierra Sur de Oaxaca, México. Participantes. Estudiantes universitarios de diferentes licenciaturas que expresaron tener una vida sexual activa. Intervenciones. Se construyó un cuestionario de respuesta cerrada que se aplicó de manera autoadministrada a los universitarios. Medidas de resultados. El instrumento fue dividido en los apartados sociodemográficos, académicos, aspectos sexuales, conocimiento sobre el VPH y prácticas de prevención sobre el virus. Resultados. Los participantes masculinos iniciaron su vida sexual más temprano que las mujeres $\left(x^{2}=25,7 ; p=0,048\right)$ y tuvieron un mayor número de parejas $\left(x^{2}=34,09\right.$ $p=0,002)$. Cerca de la mitad no utilizaba el preservativo durante sus relaciones sexuales. Desconocian que el virus está relacionado al cáncer cervicouterino $\left(x^{2}=17,5 ; p<0,001\right)$ y ambos géneros ignoraban que el VPH puede provocar cáncer en el hombre $\left(x^{2}=0,09\right.$, $p=0,760)$, tampoco conocian las principales manifestaciones clínicas de la enfermedad $\left(x^{2}=1,7 ; p=0,415\right)$. Los estudiantes del área de la salud tuvieron un mayor conocimiento en el diagnóstico del VPH. La edad no influyó en el conocimiento del virus. Conclusiones. E presente estudio reveló que la mayor parte de los estudiantes desconocía con certeza la clínica, manejo y tratamiento de la infección y como consecuencia estuvieron inadvertidos ante la importancia del contagio. Es necesario otorgar mayor importancia a la educación sexual de los universitarios y concientizarlos sobre las repercusiones que conlleva la infección del VPH, divulgando los mecanismos de transmisión, métodos de prevención y características clínicas.
\end{abstract}

Palabras clave. Conducta de riesgo; Enfermedad de transmisión sexual; Educación sexual.

\section{Abstract}

Introduction. Human papillomavirus (HPV) causes the world's most important sexually transmitted infection and is considered the main agent for cervical cancer. Youngsters are most vulnerable as they usually begin sexual relations without protection. Objectives. To describe students' knowledge of human papillomavirus infection prevention at Sierra Sur, Oaxaca University. Design. Quantitative, descriptive cross-sectional study. Place. Sierra Sur, Oaxaca, Mexico. Participants. University students from different programs who reported to have an active sexual life. Interventions. A closed response questionnaire was constructed and applied to the students by self-administration. Main outcome measures. The instrument was divided into sections including sociodemographic, academic and sexual aspects, knowledge about HPV and prevention practices. Results. Male participants became sexually active earlier than women $\left(x^{2}=25.7, p=0.048\right)$, had more partners $\left(x^{2}=34.09, p=0.002\right)$, and about half of them did not use condom during sex. They were unaware that the virus is associated with cervical cancer $\left(x^{2}=17.5, p<0.001\right)$ and may cause cancer in men $\left(x^{2}=0.09, p=0.760\right)$. They did not know the main clinical manifestations of the disease $\left(x^{2}=1.7, p=0.415\right)$. Students of the health sciences had some knowledge on HPV diagnosis. Age did not affect knowledge on the virus. Conclusions. This study revealed that most students do not know the clinical features, management and treatment of the HPV infection and as a consequence are unaware of the importance of the transmission. It is necessary to give more importance to the sex education of university students and to raise awareness of the impact of the HPV infection, by informing about the transmission mechanisms, prevention methods, and clinical manifestations.

Keywords. Risk-taking; Sexually transmitted diseases; Sex education.

An Fac med. 2015;76(4):369-76 / http://dx.doi.org/10.15381/anales.v76i4.11406

\section{INTRODUCCIÓN}

Las infecciones de transmisión sexual (ITS) representan un grave problema de salud pública a nivel mundial, que conlleva a múltiples enfermedades que pueden ocasionar la muerte ${ }^{(1)}$. La infección originada por el virus del papi- loma humano (VPH) es la enfermedad viral de transmisión sexual más frecuente y la primera causa de consulta ginecológica ${ }^{(2)}$. Se considera que todo individuo sexualmente activo estará expuesto en algún momento de su vida a este agente patógeno ${ }^{(3)}$.
El VPH pertenece a la subfamilia $\mathrm{Pa}$ pilomaviridae. El virus se caracteriza por un tropismo cutaneomucoso e infecta a células superficiales de la epidermis, y tras un microtraumatismo penetra a capas profundas, multiplicándose policlonalmente ${ }^{(4)}$. El agente viral ocasio- 
na lesiones verrugosas, bien sea en forma benigna o maligna, aunque también puede encontrarse en forma latente cuando el papilomavirus persiste en las células huésped sin manifestación clínica aparente; el período de incubación es de 2 a 3 meses o de varios años ${ }^{(5,6)}$.

Existen diferentes tipos de VPH que pueden producir el papiloma oral, papiloma laríngeo y algunos otros el papiloma en la conjuntiva y nasal (7), aunque los de mayor importancia epidemiológica son aquellos que ocasionan verrugas en el cuello del útero, vagina, uretra, pene y ano. Asimismo, aquellos que se encuentran íntimamente relacionados al desarrollo del cáncer cervicouterino, etiología que ocupa el segundo lugar de los tumores malignos en todo el mundo por su altas tasas de incidencia y prevalencia en morbimortalidad ${ }^{(8)}$. El contagio más común ocurre por contacto del virus con alguna lesión relacionada durante el acto sexual vaginal, anal u oral ${ }^{(9)}$.

Dada la transmisión, los factores de riesgo para que un individuo adquiera la infección se encuentran relacionados, aunque no son exclusivos con las ITS. Influyen además el entorno educativo y socioeconómico, la drogadicción y el tabaquismo, a lo que se suma la falta de higiene, el inicio temprano de la vida sexual, el antecedente de haber tenido sexo con dos o más parejas sin protección y, en el caso de la mujer, la edad temprana del primer embarazo, tres o más partos, el uso prolongado de anticonceptivos hormonales, además de la coexistencia de otro tipo de enfermedades que alteran la capacidad de respuesta de su sistema inmune ${ }^{(10)}$. En lo que respecta al sexo masculino, este actúa como reservorio del virus, pues rara vez presenta manifestaciones clínicas u ocasiona problemas severos de salud, lo cual impide un diagnóstico preciso y oportuno, favoreciendo la diseminación del papilomavirus ${ }^{(11)}$. La población más vulnerable de adquirir la infección son los jóvenes entre 15 y 24 años de edad, debido al inicio de las relaciones sexuales usualmente sin métodos de protección contra el VPH, así como el desconocimiento sobre las consecuencias de la infección ${ }^{(12,13)}$.

La Organización Mundial de la Salud (1) estima que $80 \%$ de la población mundial sexualmente activa adquirirá al menos una vez en su vida la infección. Asimismo, se reportan $500 \mathrm{mil}$ nuevos casos de cáncer cervicouterino al año, provocando 260 mil muertes por esta enfermedad. En México ${ }^{(14)}$ existen alrededor de 25 millones de mujeres infectadas por el VPH y anualmente se reportan 68000 nuevos casos de cáncer cervicouterino, con una incidencia de 10,06 en mujeres de 15 años o más y, de acuerdo al Instituto Nacional de Estadística y Geografía, los estados con mayores cifras son Veracruz $(25,28)$, Coahuila $(19,56)$ y Oaxaca $(15,25)$.

Para que la prevención sea eficaz, es necesaria la detección temprana y tratamiento oportuno de las lesiones; pero también es fundamental proporcionar a la población vulnerable información sobre los principales aspectos epidemiológicos, clínicos y terapéuticos de la enfermedad. Derivado de lo anterior, el objetivo de este estudio fue describir el conocimiento para la prevención del virus del papiloma humano en universitarios de la sierra sur, Oaxaca, con la finalidad de proponer estrategias preventivas que favorezcan hábitos de vida saludable en los jóvenes.

\section{MÉTODOS}

Se realizó un estudio descriptivo de corte transversal durante los meses de febrero a julio de 2013 en estudiantes de la Universidad de la Sierra Sur, institución educativa ubicada en el Estado de Oaxaca, que atiende aproximadamente al 18\% de los universitarios que pertenecen al sistema de universidades estatales de Oaxaca ${ }^{(15)}$.

La muestra se calculó considerando la matrícula de 1330 alumnos, 50\% de proporción, $5 \%$ de error muestral y 95\% de nivel de confianza. El muestreo fue de tipo probabilístico, estratificado de acuerdo a la distribución de alumnos por licenciatura: Enfermería (50\%),
Nutrición (23,7\%), Administración Pública (7,9\%), Informática (7,2\%), Ciencias Empresariales (6,5\%) y Administración Municipal (4,7\%).

Se construyó un cuestionario según los criterios propuestos por Ortunio y $\mathrm{col}^{(13)}$. El instrumento fue dividido en los siguientes apartados: a) sociodemográficos: edad, sexo, religión, estado civil y región de procedencia; b) académicos: semestre y licenciatura; c) información sobre aspectos sexuales: orientación sexual, edad de primera relación sexual y número de parejas sexuales; d) conocimiento sobre el VPH: información respecto a factores de riesgo, transmisión, manifestaciones clínicas, diagnóstico, tratamiento y prevención; y, e) prácticas de prevención sobre el VPH: prácticas de conducta sexual, accesibilidad a información y revisión médica periódica.

Para estandarizar el instrumento de medición, se efectuó la prueba en algunos participantes, con la posterior revisión por los especialistas. Para realizar el estudio se obtuvo la autorización de las autoridades universitarias y de los participantes a través del consentimiento informado, previa explicación del objetivo de la investigación, aclarando las dudas respecto del proyecto. A continuación, de los universitarios que expresaron haber iniciado una vida sexual se les aplicó la encuesta de manera autoadministrada, siempre con la presencia del investigador para resolver alguna duda durante el llenado del cuestionario.

En la recolección de información se construyó una base de datos con el programa Excel Microsoft 2007, según el género, tipo de licenciatura y el semestre en que los participantes estaban adscritos. Para el análisis descriptivo se empleó las medidas de tendencia central y distribución de frecuencias. En cuanto al estudio inferencial, se ejecutó la prueba de chi cuadrado para establecer diferencia significativa entre las variables. Se utilizó el programa estadístico Sigma Stat versión 3.1, con un nivel de significancia de $\alpha=0,05$. 


\section{RESULTADOS}

La edad de los entrevistados osciló entre 18 y 26 años, con promedio de $20,7 \pm 2,1$, y predominó el género femenino $(61,4 \%)$. En lo que se refiere al estado civil, 89,9\% declaró ser soltero, $6,9 \%$ en unión libre y 3,2\% casado. La religión que predominó fue la católica $(81 \%)$, luego la cristiana $(9,7 \%)$ y otras $(9,3 \%)$. Por región de procedencia, la mayor parte de los alumnos procedían de la Sierra Sur $(31,2 \%)$, seguido de Valles Centrales (29,3\%), Costa $(21,1 \%)$, Istmo (5,9\%), Mixteca (5,2\%), Sierra Norte (3\%), otros Estados (2,6\%) y de otras zonas de Oaxaca (1,4\%).

Dado el ciclo escolar en que se llevó a cabo la investigación, el semestre con mayor número de participantes fue el segundo $(38,1 \%)$, a continuación el cuarto $(23,7 \%)$, octavo $(20,5 \%)$, sexto $(14,0 \%)$ y décimo $(3,6 \%)$. En cuanto a la orientación sexual, el 93,2\% comentó ser heterosexual, 4,1\% homosexual y $2,6 \%$ bisexual.

En la tabla 1 se presenta información sobre los aspectos sexuales de los participantes. Destaca que en los hombres existió una discreta aunque significativa diferencia $\left(\chi^{2}=25,7 ; p=0,048\right)$ en el inicio de la vida sexual en comparación con las mujeres; de igual manera, los hombres tuvieron mayor número de parejas sexuales que las mujeres $\left(\chi^{2}=34,09 ; p=0,002\right)$. Cuando se compara los aspectos sexuales por licenciatura, no existió diferencia en el inicio de la vida sexual $\left(\chi^{2}=92 ; p=0,088\right)$ y el número de parejas $\left(\chi^{2}=90,3\right.$; $\mathrm{p}=0,051)$; en cambio, los jóvenes del décimo semestre comentaron un inicio de la vida sexual más precoz en comparación con los demás semestres $\left(\chi^{2}=166,68 ; \mathrm{p}<0,001\right)$.

En cuanto al conocimiento sobre el papilomavirus (tabla 2), se encontró que los hombres desconocían métodos de prevención de la infección $\left(\chi^{2}=4,5\right.$; $\mathrm{p}=0,034)$, así también lo que el virus provoca en las mujeres $\left(\chi^{2}=17,5\right.$; $\mathrm{p}<0,001)$, utilidad de la prueba del Papanicolaou $\quad\left(\chi^{2}=22,2 ; \quad \mathrm{p}<0,001\right)$, existencia de la vacuna que previniera

Tabla 1. Aspectos sexuales de los universitarios.

\begin{tabular}{|c|c|c|c|}
\hline & & $\begin{array}{l}\text { Edad primera relación } \\
\text { sexual } \\
\text { Media (desviación estándar) }\end{array}$ & $\begin{array}{l}\text { Número de parejas } \\
\text { sexuales } \\
\text { Intervalo (mediana) }\end{array}$ \\
\hline \multicolumn{2}{|l|}{ General } & $17,9(2,4)$ & 1 a $18(2)$ \\
\hline \multirow{2}{*}{ Por género } & Hombres & $17,5(2,4)^{a}$ & 1 a $18(4)^{a}$ \\
\hline & Mujeres & $18,1(2,4)^{b}$ & 1 a $10(1)^{b}$ \\
\hline \multirow{6}{*}{ Por licenciatura } & Enfermería & $17,8(2,0)^{\mathrm{a}}$ & 1 a $18(2)^{\mathrm{a}}$ \\
\hline & Nutrición & $18,3(2,5)^{\mathrm{a}}$ & 1 a $16(2)^{a}$ \\
\hline & Informática & $18,2(2,1)^{\mathrm{a}}$ & 1 a $5(2)^{a}$ \\
\hline & Administración pública & $17,6(2,2)^{\mathrm{a}}$ & 1 a $9(3)^{\text {a }}$ \\
\hline & Administración municipal & $18,4(2,5)^{\mathrm{a}}$ & 1 a $10(4)^{\mathrm{a}}$ \\
\hline & Ciencias empresariales & $16,6(4,2)^{\mathrm{a}}$ & 1 a $11(2)^{\mathrm{a}}$ \\
\hline \multirow{5}{*}{ Por semestre } & Segundo semestre & $17,1(1,7)^{\mathrm{b}}$ & 1 a $18(2)^{a}$ \\
\hline & Cuarto semestre & $18,0(2,2)^{\mathrm{b}}$ & 1 a $12(2)^{a}$ \\
\hline & Sexto semestre & $18,9(2,6)^{\mathrm{b}}$ & 1 a $14(2)^{\mathrm{a}}$ \\
\hline & Octavo semestre & $18,6(1,9)^{b}$ & 1 a $12(2)^{\mathrm{a}}$ \\
\hline & Decimo semestre & $16,4(6,3)^{\mathrm{a}}$ & 1 a $11(2)^{a}$ \\
\hline
\end{tabular}

Letras iguales: no existió diferencia significativa $p>0,05$.

la infección de VPH $\left(\chi^{2}=18 ; \mathrm{p}<0,001\right)$ y la edad recomendada para la vacunación $\left(\chi^{2}=11,1 ; p=0,004\right)$. Aunque ambos géneros comentaron que tener múltiples parejas sexuales es un factor de riesgo para adquirir la enfermedad $\left(\chi^{2}=0,2 ; p=0,895\right)$, desconocían las principales manifestaciones clínicas $\left(\chi^{2}=1,7 ; \quad p=0,415\right), \quad$ la posibilidad que el virus puede provocar cáncer en el hombre $\left(\chi^{2}=0,09 ; p=0,760\right)$ y los planes de manejo y tratamiento para evitar complicaciones por VPH $\left(\chi^{2}=1,9 ; \mathrm{p}=0,167\right)$. Cuando se analizó la información por licenciaturas, los estudiantes del área de la salud expresaron que el virus solo afecta a las mujeres $\left(\chi^{2}=37,9 ; \mathrm{p}<0,001\right)$, es un agente relacionado al cáncer cervicouterino $\left(\chi^{2}=36,8 ; p<0,001\right)$, conocen sobre métodos de detección del VPH $\left(\chi^{2}=26,1 ; p<0,001\right)$ y expresaron que existe una vacuna contra el agente viral $\left(\chi^{2}=73,5 ; p<0,001\right)$. En el caso de los estudiantes de la licenciatura en enfermería, conocían sobre la edad recomendada para la vacunación $\left(\chi^{2}=65,6 ; p<0,001\right)$. Sin embargo, en otros rubros, todos los estudiantes poseían conocimiento sobre la prin- cipal forma de transmisión del virus $\left(\chi^{2}=4,3 ; p=0,506\right)$, pero carecían de información referente a las manifestaciones clínicas $\left(\chi^{2}=34 ; p=0,106\right)$, la posibilidad que el virus puede provocar cáncer en el hombre $\left(\chi^{2}=0,09\right.$; $\mathrm{p}=0,760)$ y los planes de manejo y tratamiento para evitar complicaciones por $\operatorname{VPH}\left(\chi^{2}=9,2 ; \mathrm{p}=0,101\right)$.

Por otro lado, cuando la información se analiza por semestre, no existió diferencia significativa $(\mathrm{p}>0,05)$, es decir, en todas las edades, carecían de información sobre mecanismos de transmisión, manifestaciones clínicas, métodos de diagnóstico y planes de manejo y tratamiento para evitar complicaciones, e incluso en algunos rubros como aspectos relacionados a la vacunación el conocimiento fue deficiente $\left(\chi^{2}=17,29 ; p=0,027\right)$.

En lo que corresponde a las prácticas de prevención (tabla 3), independientemente si se analiza por género, licenciatura o por semestre, alrededor de $40 \%$ de los encuestados comentó utilizar preservativo durante las relaciones sexuales imprevistas o con una pareja estable (por género $\chi^{2}=7,8 ; p=0,247$, 
Tabla 2. Conocimiento sobre el virus del papiloma humano de los universitarios (porcentaje de respuestas correctas).

\begin{tabular}{|c|c|c|c|c|c|c|c|c|c|c|c|c|c|c|}
\hline & & 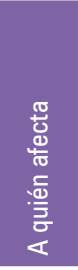 & 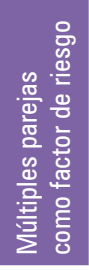 & 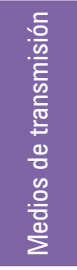 & 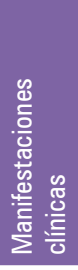 & 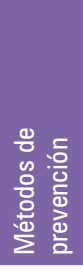 & 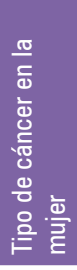 & 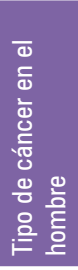 & 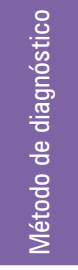 & 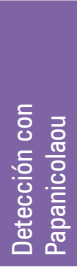 & 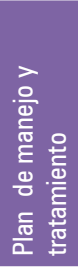 & 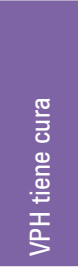 & 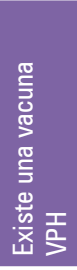 & 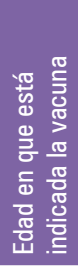 \\
\hline General & & 60,7 & 96,7 & 93,4 & 37,7 & 84,9 & 82,8 & 45,3 & 62,4 & 80,9 & 10,0 & 22,7 & 56,3 & 32,2 \\
\hline \multirow[t]{2}{*}{ Por género } & Hombres & $67,3^{\mathrm{a}}$ & $96,3^{\mathrm{a}}$ & $96,2^{\mathrm{a}}$ & $34,4^{\mathrm{a}}$ & $78,7^{\mathrm{b}}$ & $70,4^{b}$ & $43,5^{\mathrm{a}}$ & $59,4^{b}$ & $68,2^{b}$ & $6,5^{\mathrm{a}}$ & $25,0^{\mathrm{a}}$ & $40,7^{\mathrm{b}}$ & $20,6^{b}$ \\
\hline & Mujeres & $56,2^{\mathrm{a}}$ & $97^{\mathrm{a}}$ & $91,7^{\mathrm{a}}$ & $39,5^{\mathrm{a}}$ & $88,8^{\mathrm{a}}$ & $90,6^{\mathrm{a}}$ & $46,2^{\mathrm{a}}$ & $63,8^{\mathrm{a}}$ & $89,3^{\mathrm{a}}$ & $12,4^{\mathrm{a}}$ & $21,3^{\mathrm{a}}$ & $66,5^{\mathrm{a}}$ & $39,9^{a}$ \\
\hline \multirow[t]{6}{*}{ Por licenciatura } & Enfermería & $50,7^{a}$ & $97,1^{\mathrm{b}}$ & $92,7^{\mathrm{a}}$ & $38,4^{\mathrm{a}}$ & $31,4^{b}$ & $92,1^{\text {a }}$ & $44,9^{\mathrm{a}}$ & $80,0^{\mathrm{a}}$ & $90,6^{a}$ & $15,1^{\mathrm{a}}$ & $21,7^{\mathrm{a}}$ & $74,1^{\mathrm{a}}$ & $54,0^{\mathrm{a}}$ \\
\hline & Nutrición & $63,6^{\mathrm{a}}$ & $100^{\mathrm{a}}$ & $98,5^{\mathrm{a}}$ & $39,3^{\mathrm{a}}$ & $88,1^{\mathrm{a}}$ & $83,6^{\mathrm{a}}$ & $46,3^{\mathrm{a}}$ & $74,0^{\mathrm{a}}$ & $83,3^{\mathrm{a}}$ & $4,5^{\mathrm{a}}$ & $26,9^{a}$ & $56,7^{\mathrm{a}}$ & $16,4^{\mathrm{b}}$ \\
\hline & Informática & $65,0^{\mathrm{a}}$ & $100^{\mathrm{b}}$ & $100^{a}$ & $22,2^{\mathrm{a}}$ & $70,0^{\mathrm{a}}$ & $55,0^{\mathrm{b}}$ & $45,0^{\mathrm{a}}$ & $55,6^{b}$ & $55,0^{\mathrm{b}}$ & $0,0^{\mathrm{a}}$ & $15,0^{\mathrm{a}}$ & $20,2^{b}$ & $0,0^{\mathrm{b}}$ \\
\hline & Administración pública & $81,0^{\mathrm{b}}$ & $81,8^{\mathrm{a}}$ & $72,2^{\mathrm{a}}$ & $35,0^{\mathrm{a}}$ & $77,3^{\mathrm{a}}$ & $68,2^{b}$ & $40,9^{\mathrm{a}}$ & $13,6^{b}$ & $68,2^{\mathrm{b}}$ & $4,5^{\mathrm{a}}$ & $27,3^{\mathrm{a}}$ & $18,2^{\mathrm{b}}$ & $9,5^{b}$ \\
\hline & Administración municipal & $84,6^{b}$ & $100^{\mathrm{b}}$ & $100^{\mathrm{a}}$ & $36,4^{\mathrm{a}}$ & $46,2^{b}$ & $92,3^{\mathrm{a}}$ & $30,8^{\mathrm{a}}$ & $75,0^{\mathrm{a}}$ & $61,5^{b}$ & $7,7^{\mathrm{a}}$ & $15,4^{\mathrm{a}}$ & $38,5^{b}$ & $15,4^{\mathrm{b}}$ \\
\hline & Ciencias empresariales & $83,3^{b}$ & $94,4^{\mathrm{b}}$ & $88,9^{\mathrm{a}}$ & $37,5^{\mathrm{a}}$ & $77,8^{\mathrm{a}}$ & $50,0^{\mathrm{b}}$ & $61,1^{\mathrm{a}}$ & $65,5^{b}$ & $55,6^{b}$ & $11,1^{\mathrm{a}}$ & $22,2^{\mathrm{a}}$ & $16,7^{\mathrm{b}}$ & $0,0^{\mathrm{b}}$ \\
\hline \multirow[t]{5}{*}{ Por semestre } & Segundo & $59,0^{a}$ & $96,2^{\mathrm{a}}$ & $93,3^{\mathrm{a}}$ & $35,8^{\mathrm{a}}$ & $80,4^{\mathrm{a}}$ & $80,4^{\mathrm{a}}$ & $48,1^{\mathrm{a}}$ & $77,4^{\mathrm{a}}$ & $60,7^{\mathrm{a}}$ & $8,7^{\mathrm{a}}$ & $40,6^{a}$ & $60,7^{\mathrm{a}}$ & $21,7^{\mathrm{a}}$ \\
\hline & Cuarto & $50,0^{\mathrm{a}}$ & $93,9^{\mathrm{a}}$ & $96,9^{a}$ & $43,3^{\mathrm{a}}$ & $90,9^{a}$ & $83,3^{\mathrm{a}}$ & $43,9^{a}$ & $80,3^{\mathrm{a}}$ & $60,0^{\mathrm{a}}$ & $12,1^{\mathrm{a}}$ & $50,0^{\mathrm{a}}$ & $66,7^{\mathrm{a}}$ & $40,6^{\mathrm{a}}$ \\
\hline & Sexto & $53,8^{\mathrm{a}}$ & $100^{\mathrm{a}}$ & $94,9^{\mathrm{a}}$ & $37,8^{\mathrm{a}}$ & $82,1^{\mathrm{a}}$ & $82,1^{\mathrm{a}}$ & $46,2^{\mathrm{a}}$ & $82,1^{\mathrm{a}}$ & $66,7^{\mathrm{a}}$ & $10,3^{\mathrm{a}}$ & $38,5^{\mathrm{a}}$ & $56,4^{\mathrm{a}}$ & $46,2^{\mathrm{a}}$ \\
\hline & Octavo & $78,6^{\mathrm{a}}$ & $100^{\mathrm{a}}$ & $91,1^{\text {a }}$ & $36,8^{\mathrm{a}}$ & $91,2^{\mathrm{a}}$ & $86,0^{\mathrm{a}}$ & $42,1^{\mathrm{a}}$ & $89,3^{\mathrm{a}}$ & $64,2^{\mathrm{a}}$ & $19,3^{b}$ & $61,4^{\mathrm{a}}$ & $68,4^{\mathrm{a}}$ & $36,6^{a}$ \\
\hline & Decimo & $80,0^{\mathrm{a}}$ & $90,0^{\mathrm{a}}$ & $100^{\mathrm{a}}$ & $22,2^{\mathrm{a}}$ & $70,0^{\mathrm{a}}$ & $90,0^{\mathrm{a}}$ & $90,0^{\mathrm{a}}$ & $70,0^{\mathrm{a}}$ & $75,0^{\mathrm{a}}$ & $10,0^{\mathrm{a}}$ & $30,0^{\mathrm{a}}$ & $20,0^{\mathrm{b}}$ & $0,0^{\mathrm{b}}$ \\
\hline
\end{tabular}

Letras iguales: no existe diferencia significativa $p>0,05$.

Tabla 3. Prácticas de prevención sobre el virus del papiloma humano de los universitarios (porcentaje de respuestas que corresponden a 'siempre' y 'nunca').

\begin{tabular}{|c|c|c|c|c|c|c|c|c|c|c|c|c|c|c|c|c|c|}
\hline & \multicolumn{2}{|c|}{ 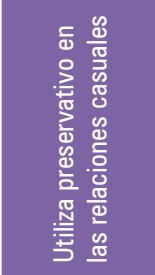 } & \multicolumn{2}{|c|}{ 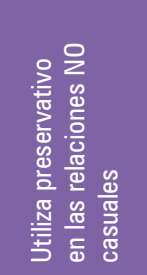 } & \multicolumn{2}{|c|}{ 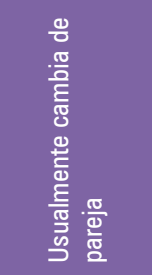 } & \multicolumn{2}{|c|}{ 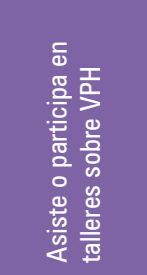 } & \multicolumn{2}{|c|}{ 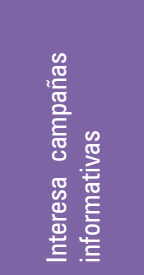 } & \multicolumn{2}{|c|}{ 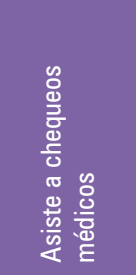 } & \multicolumn{2}{|c|}{ 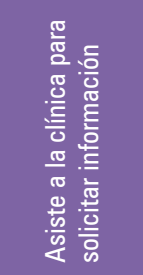 } & \multicolumn{2}{|c|}{ 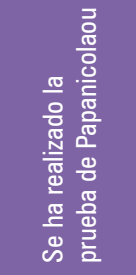 } \\
\hline & & 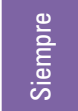 & $\begin{array}{l}\text { J } \\
\text { E }\end{array}$ & $\begin{array}{l}\frac{\infty}{2} \\
\frac{\bar{E}}{\omega} \\
\frac{\omega}{\infty}\end{array}$ & $\begin{array}{l}\mathbb{J} \\
\text { E } \\
\text { E }\end{array}$ & $\begin{array}{l}\frac{0}{2} \\
\frac{\bar{E}}{\omega} \\
\frac{\omega}{\infty}\end{array}$ & $\begin{array}{l}\stackrel{\mathbb{J}}{E} \\
\text { E }\end{array}$ & 袬 & $\begin{array}{l}\text { J } \\
\text { E }\end{array}$ & ळ. & 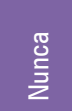 & 产 & 造 & 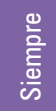 & $\begin{array}{l}\text { Ð } \\
\text { E }\end{array}$ & 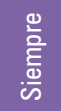 & $\begin{array}{l}\text { ల } \\
\text { E }\end{array}$ \\
\hline General & & 41,2 & 9,6 & 40,1 & 8,9 & 1,1 & 49,3 & 0,7 & 50,2 & 0,7 & 48,4 & 1,8 & 41,9 & 0,4 & 73,8 & 1,7 & 80,0 \\
\hline \multirow[t]{2}{*}{ Por género } & Hombres & $43,6^{\mathrm{a}}$ & $5,0^{\mathrm{a}}$ & $44,2^{\mathrm{a}}$ & $5,8^{\mathrm{a}}$ & $0,9^{\mathrm{a}}$ & $34,9^{b}$ & $0,0^{\mathrm{a}}$ & $15,0^{\mathrm{a}}$ & $0,0^{\mathrm{a}}$ & $56,1^{\mathrm{a}}$ & $0,0^{\mathrm{a}}$ & $50,5^{b}$ & $0,9^{a}$ & $52,3^{\mathrm{a}}$ & - & - \\
\hline & Mujeres & $39,6^{\mathrm{a}}$ & $12,6^{\mathrm{a}}$ & $37,0^{\mathrm{a}}$ & $10,9^{a}$ & $1,2^{\mathrm{a}}$ & $58,8^{\mathrm{a}}$ & $4,1^{\mathrm{a}}$ & $10,0^{\mathrm{a}}$ & $1,2^{\mathrm{a}}$ & $46,5^{\mathrm{a}}$ & $2,9^{\mathrm{a}}$ & $36,5^{\mathrm{a}}$ & $0,6^{\mathrm{a}}$ & $45,3^{\mathrm{a}}$ & 1,8 & 79,9 \\
\hline \multirow[t]{6}{*}{ Por licenciatura } & Enfermería & $40,2^{\mathrm{a}}$ & $11,4^{\mathrm{a}}$ & $43,7^{\mathrm{a}}$ & $8,1^{\mathrm{a}}$ & $0,7^{\mathrm{a}}$ & $56,1^{\mathrm{a}}$ & $2,9^{a}$ & $5,8^{\mathrm{a}}$ & $1,4^{\mathrm{a}}$ & $46,8^{a}$ & $2,2^{\mathrm{a}}$ & $41,7^{\mathrm{a}}$ & $0,7^{\mathrm{a}}$ & $38,1^{b}$ & $2,8^{a}$ & $80,7^{\mathrm{a}}$ \\
\hline & Nutrición & $39,1^{\mathrm{a}}$ & $4,7^{\mathrm{a}}$ & $29,2^{\mathrm{a}}$ & $13,8^{\mathrm{a}}$ & $1,5^{\mathrm{a}}$ & $49,3^{\mathrm{a}}$ & $4,5^{\mathrm{a}}$ & $13,4^{\mathrm{a}}$ & $0,0^{\mathrm{a}}$ & $50,7^{\mathrm{a}}$ & $3,0^{\mathrm{a}}$ & $35,8^{b}$ & $1,5^{\mathrm{a}}$ & $56,7^{\mathrm{b}}$ & $0,0^{\mathrm{a}}$ & $83,7^{\mathrm{a}}$ \\
\hline & Informática & $44,4^{a}$ & $5,6^{\mathrm{a}}$ & $63,2^{\mathrm{a}}$ & $10,5^{\mathrm{a}}$ & $0,0^{\mathrm{a}}$ & $26,3^{b}$ & $0,0^{\mathrm{a}}$ & $10,0^{\mathrm{a}}$ & $0,0^{\mathrm{a}}$ & $45,0^{\mathrm{a}}$ & $0,0^{\mathrm{a}}$ & $55,0^{\mathrm{a}}$ & $0,0^{\mathrm{a}}$ & $45,0^{\mathrm{b}}$ & $0,0^{\mathrm{a}}$ & $50,0^{\mathrm{b}}$ \\
\hline & Administración pública & $57,9^{\mathrm{a}}$ & $5,3^{\mathrm{a}}$ & $36,4^{\mathrm{a}}$ & $0,0^{\mathrm{a}}$ & $0,0^{\mathrm{a}}$ & $45,5^{\mathrm{a}}$ & $0,0^{\mathrm{a}}$ & $22,7^{\mathrm{b}}$ & $0,0^{\mathrm{a}}$ & $50,0^{\mathrm{a}}$ & $0,0^{\mathrm{a}}$ & $40,9^{\mathrm{a}}$ & $0,0^{\mathrm{a}}$ & $68,2^{\mathrm{a}}$ & $0,0^{\mathrm{a}}$ & $55,6^{b}$ \\
\hline & Administración municipal & $25,0^{\mathrm{a}}$ & $25,0^{\mathrm{a}}$ & $25,0^{\mathrm{a}}$ & $0,0^{\mathrm{a}}$ & $7,7^{\mathrm{a}}$ & $53,8^{\mathrm{a}}$ & $0,0^{\mathrm{a}}$ & $30,8^{b}$ & $0,0^{\mathrm{a}}$ & $76,9^{\mathrm{b}}$ & $0,0^{\mathrm{a}}$ & $23,1^{b}$ & $0,0^{\mathrm{a}}$ & $84,6^{\mathrm{a}}$ & 0,0 & $71,4^{\mathrm{a}}$ \\
\hline & Ciencias empresariales & $43,0^{\mathrm{a}}$ & $12,5^{\mathrm{a}}$ & $41,2^{\mathrm{a}}$ & $11,8^{\mathrm{a}}$ & $0,0^{\mathrm{a}}$ & $47,1^{\mathrm{a}}$ & $0,0^{\mathrm{a}}$ & $35,8^{\mathrm{b}}$ & $0,0^{\mathrm{a}}$ & $64,7^{\mathrm{a}}$ & $0,0^{\mathrm{a}}$ & $47,1^{\mathrm{a}}$ & $0,0^{\mathrm{a}}$ & $58,8^{\mathrm{b}}$ & $0,0^{\mathrm{a}}$ & $100^{\mathrm{a}}$ \\
\hline \multirow[t]{5}{*}{ Por semestre } & Segundo & $39,4^{\mathrm{a}}$ & $10,1^{\mathrm{a}}$ & $45,1^{\mathrm{a}}$ & $9,8^{\mathrm{a}}$ & $0,0^{\mathrm{a}}$ & $54,7^{\mathrm{a}}$ & $0,0^{\mathrm{a}}$ & $54,2^{\mathrm{a}}$ & $0,0^{\mathrm{a}}$ & $45,8^{\mathrm{a}}$ & $2,8^{\mathrm{a}}$ & $42,1^{\mathrm{a}}$ & $0,0^{\mathrm{a}}$ & $83,2^{\mathrm{a}}$ & $1,5^{\mathrm{a}}$ & $88,1^{\mathrm{a}}$ \\
\hline & Cuarto & $38,3^{\mathrm{a}}$ & $6,7^{\mathrm{a}}$ & $31,8^{\mathrm{a}}$ & $10,6^{\mathrm{a}}$ & $0,0^{\mathrm{a}}$ & $47,0^{\mathrm{a}}$ & $0,0^{\mathrm{a}}$ & $47,0^{\mathrm{a}}$ & $1,5^{\mathrm{a}}$ & $51,5^{\mathrm{a}}$ & $1,5^{\mathrm{a}}$ & $45,5^{\mathrm{a}}$ & $0,0^{a}$ & $63,6^{\mathrm{a}}$ & $0,0^{\mathrm{a}}$ & $81,0^{\mathrm{a}}$ \\
\hline & Sexto & $51,3^{\mathrm{a}}$ & $5,1^{\mathrm{a}}$ & $50,0^{\mathrm{a}}$ & $2,6^{\mathrm{a}}$ & $7,7^{\mathrm{a}}$ & $38,5^{\mathrm{a}}$ & $2,6^{\mathrm{a}}$ & $42,6^{\mathrm{a}}$ & $0,0^{\mathrm{a}}$ & $46,3^{\mathrm{a}}$ & $0,0^{\mathrm{a}}$ & $33,3^{\mathrm{a}}$ & $0,0^{\mathrm{a}}$ & $59,0^{\mathrm{a}}$ & $4,2^{\mathrm{a}}$ & $62,5^{\mathrm{a}}$ \\
\hline & Octavo & $40,7^{\mathrm{a}}$ & $9,3^{\mathrm{a}}$ & $35,2^{\mathrm{a}}$ & $9,3^{\mathrm{a}}$ & $0,0^{\mathrm{a}}$ & $48,2^{\mathrm{a}}$ & $1,8^{\mathrm{a}}$ & $46,2^{\mathrm{a}}$ & $1,8^{\mathrm{a}}$ & $41,2^{\mathrm{a}}$ & $1,8^{\mathrm{a}}$ & $39,3^{\mathrm{a}}$ & $1,9^{a}$ & $74,1^{\mathrm{a}}$ & $5,1^{\text {a }}$ & $74,4^{a}$ \\
\hline & Decimo & $33,3^{\mathrm{a}}$ & $44,4^{\mathrm{a}}$ & $33,3^{\mathrm{a}}$ & $44,4^{a}$ & $30,0^{\mathrm{a}}$ & $10,0^{\mathrm{b}}$ & $0,0^{\mathrm{a}}$ & $70,0^{\mathrm{a}}$ & $0,0^{\mathrm{a}}$ & $70,0^{\mathrm{a}}$ & $0,0^{\mathrm{a}}$ & $60,0^{b}$ & $0,0^{\mathrm{a}}$ & $100^{\mathrm{b}}$ & $0,0^{\mathrm{a}}$ & $100^{b}$ \\
\hline
\end{tabular}

Letras iguales: no existe diferencia significativa $p>0,05$. 
por licenciaturas $s^{2}=39,18 ; \mathrm{p}=0,122$ y por semestre $\left.\chi^{2}=12,75 ; \mathrm{p}=0,888\right)$. No obstante, los hombres tendieron a cambiar de pareja $\left(\chi^{2}=27,4 ; \mathrm{p}<0,001\right)$ independientemente del tipo de licenciatura $\left(\chi^{2}=26 ; p=0,165\right)$ y con mayor frecuencia en el décimo semestre $\left(\chi^{2}=28,3 ; p=0,029\right)$. Los estudiantes no asistían o participaban en talleres, simposios, congresos (por género $\chi^{2}=7,3 ; \mathrm{p}=0,120$, por licenciaturas $\chi^{2}=10,6 ; \mathrm{p}=0,995$ y por semestre $\left.\chi^{2}=8,82 ; \mathrm{p}=0,920\right)$ ni se interesaban en campañas informativas sobre el VPH (por género $\chi^{2}=4,2 ; p=0,378$; licenciaturas $\chi^{2}=26,2 ; \mathrm{p}=0,158$ y por semestre $\left.\chi^{2}=14,04 ; p=0,596\right)$. Por otra parte, las mujeres acudían a revisión médica más frecuentemente que los hombres $\left(\chi^{2}=16,7 ; p=0,002\right)$; esto sucedía por igual en todas las licenciaturas $\left(\chi^{2}=14,9 ; \mathrm{p}=0,778\right)$ excepto en las alumnas del décimo semestre $\left(\chi^{2}=29,7 ; p=0,075\right)$; destacó que la consulta médica no la efectuaba para solicitar información sobre el VPH (por género $\chi^{2}=3,8 ; p=0,576$, por licenciaturas $\left.\chi^{2}=17,8 ; \mathrm{p}=0,850\right)$ ni para realizarse la prueba de del Papanicolaou (para licenciatura $\chi^{2}=28,8 ; \mathrm{p}=0,271$ y por semestre $\left.\chi^{2}=17,69 ; \mathrm{p}=0,608\right)$.

\section{DISCUSIÓN}

Aunque diversos estudios ${ }^{(13,16,17,18)}$ en diferentes regiones del mundo, aún en países desarrollados, demuestran que los jóvenes durante la etapa universitaria carecen de conocimientos óptimos para la prevención del VPH, sin embargo, este tipo de investigaciones en el caso del estado de Oaxaca, caracterizado por entornos epidemiológicos y sociales de alta marginalidad y bajo desarrollo humano ${ }^{(19,20)}$ con población predominantemente indígena ${ }^{(21)} \mathrm{y}$ acceso insuficiente a los servicios de salud para una orientación sexual, una consulta en la utilización de métodos anticonceptivos y exámenes preventivos como el Papanicolaou son de gran trascendencia para cerrar la brecha entre las campañas de prevención y la sensibilización de los jóvenes para que acce- dan a estudios diagnósticos y de tratamiento que beneficien a su propia salud y la de su entorno. Cabe mencionar que en México, el estado de Oaxaca se encuentra entre las principales entidades con mayores tasas de mortalidad por cáncer cervicouterino y de acuerdo a García y col (22) en los últimos años se incrementó la frecuencia de casos de morbimortalidad en mujeres jóvenes por este tipo de cáncer.

Para disminuir la incidencia y la tasa de mortalidad por cáncer cervicouterino es indispensable incrementar el acceso de la población vulnerable a los programas de detección oportuna de esta enfermedad. Aunque existen pruebas moleculares de diagnóstico ${ }^{(23)}$, el alto costo hace que sea inaccesible para la mayor parte de la población y las unidades públicas de salud en México ${ }^{(24,25)}$ solo cuentan como métodos de detección la captura de híbridos, citología cervical, biopsia, colposcopia con aplicación de ácido acético y la vacunación gratuita de unidad recombinante tetravalente (tipos $6,11,16$ y 18) o bivalente (16 y 18 ), que previenen casi todas las lesiones relacionadas con el virus en mujeres no expuestas previamente. La población carece de interés sobre la enfermedad, consecuencias y medidas preventivas. Por ello, es necesario fomentar el diseño de campañas de promoción principalmente en adolescentes y jóvenes, ya que las técnicas de tamizaje y detección solo funcionarán siempre y cuando a toda edad las mujeres tengan no solamente acceso gratuito a ellas sino también cuenten con información oportuna que genere interés y conocimientos en los jóvenes respecto al VPH y a las patologías asociadas.

En este estudio, el inicio de la vida sexual de los encuestados fue alrededor de los 16 a 18 años, es decir, período que coincide con el ingreso a la formación universitaria, por lo que se considera un factor de riesgo, no por ser sexualmente activos sino porque de acuerdo a las condiciones socioculturales no cuentan con una preparación y orientación para un comportamiento responsable que haga posible generar conciencia sobre las consecuencias de incurrir en prácticas que pongan en peligro la salud; lo cual ratifica en la necesidad e importancia de la información sobre la temática sexual como una importante medida de prevención ${ }^{(13,17)}$.

Por otra parte, destaca que la mayor parte de los alumnos $(93,4 \%)$ tuvieron conocimiento sobre el principal mecanismo de transmisión del VPH, en contraste con otros universitarios $(26,27)$ de contextos urbanos que negaron tener dicha noción (menor al 50\%). De igual manera, los hombres comentaron una edad menor en el inicio de la vida sexual, que fue reflejada en el mayor número de parejas. En opinión de diversos especialistas ${ }^{(28,29)}$, a mayor número de parejas sexuales incrementa el riesgo de contagiarse por VPH. Esto tiene gran relevancia si se considera que los hombres desconocen de métodos de prevención contra el virus, análogo a lo observado por McPartland y col ${ }^{(30)}$ en universitarios norteamericanos, que manifestaron $(54,9 \%)$ desinterés en el tema. Aunado a ello, solo 78,7\% de los hombres tenía información para evitar el contagio. Dell y col ${ }^{(31)}$ expresan que el uso incorrecto del preservativo o la ausencia del mismo en las relaciones sexuales predisponen al contagio del VPH y otras ITS.

En este mismo sentido, los estudiantes no reconocen con certeza la clínica de la infección por VPH y como consecuencia, de acuerdo a lo señalado por Fox ${ }^{(32)}$, se mantienen inadvertidos ante la importancia de adquirir la infección. Por lo tanto, constituye una amenaza contra la salud pública y sexual. De ahí que la información es vital para evitar la diseminación de la infección, sobre todo considerando que la transmisión del virus involucra mecanismos de contacto con piel infectada ${ }^{(18)}$. Dell y col ${ }^{(31)}$ proponen la difusión en el uso del condón femenino debido a que aminora la probabilidad de contraer el virus, pues cubre más área genital y brinda mejor protección que el preservativo masculino. 
Los resultados relativos a la comprensión sobre el tipo de lesiones son semejantes a otros estudios ${ }^{(16,29)}$, donde la mayor parte de los encuestados desconoce las consecuencias del contagio por el virus, en especial como factor de riesgo para el desarrollo del cáncer cervicouterino y cáncer en el pene. No obstante, las mujeres tuvieron un mayor conocimiento $(89,3 \%)$ sobre la prueba de Papanicolaou respecto a otros estudios ${ }^{(16,33)}$, donde solamente $40 \%$ mostró comprensión de esta prueba.

Existe un desconocimiento sobre el plan de manejo y tratamiento para evitar complicaciones por VPH y esto cobra gran importancia porque, de acuerdo con la opinión de Herrero y col ${ }^{(34)}$, las manifestaciones se pueden curar sin ningún tratamiento, lo que incrementa la probabilidad que una persona pueda infectarse, curarse de la infección y volverse a infectar por un nuevo tipo de $\mathrm{VPH}$.

Los resultados sobre el conocimiento de una vacuna para prevenir el VPH $(56,3 \%)$ fueron superiores al de mujeres colombianas ${ }^{(35)}$ pero deficientes en comparación a otros estudios relacionados ${ }^{(33)}$, donde al menos $75 \%$ de los encuestados comenta tener información sobre la vacuna. Según Kim y Goldie ${ }^{(36)}$, los programas para prevenir el cáncer cervicouterino deben priorizar inicialmente la cobertura en la población más vulnerable, que son las jóvenes adolescentes de 10 a 14 años, a través de la información en los planteles educativos y administración de la vacuna en colaboración con los centros de salud. Pero, también debe de acompañarse con pruebas de detección oportuna en mujeres que han empezado la vida sexual.

Respecto al conocimiento sobre el diagnóstico del VPH, los estudiantes del área de la salud tuvieron mayor comprensión. Sin embargo, en otros aspectos como formas de contagio, métodos de prevención y tratamiento fueron semejantes a otras áreas, lo que coincide con Falcao y col ${ }^{(37)}$ quienes comentan que los alumnos poseen conocimientos limitados independientemente de su área; así sugieren que es necesario generar conciencia en el cuidado de la salud de las personas implementando un programa de prevención contra VPH.

De igual manera, la población femenina tuvo mayor conocimiento respecto al género masculino, lo que coincide con Agüero y col ${ }^{(38)}$ y Ortunio y col ${ }^{(13)}$, aunque contrasta con lo hallado por $\mathrm{Pi}$ cón (39) de que $90 \%$ de las encuestadas tenía conocimiento muy escaso sobre VPH. En opinión de Suarez-Allen y $\mathrm{col}^{(40)}$, lo anterior se explica debido a que la información que se proporciona sobre el VPH en medios impresos y electrónicos se enfoca a la infección en la mujer; es posible que los varones no se sienten vulnerables y, por lo tanto, no les interesa el tema; de hecho, se perciben menos susceptibles al contagio que las mujeres.

En lo que concierne a prácticas de prevención, los resultados son semejantes con los datos reportados por el consejo nacional de población ${ }^{(41)}$ en donde cerca de la mitad de los participantes no utilizan el preservativo durante sus relaciones sexuales. Esto confirma lo comentado por Hernández y col ${ }^{(26)}$ respecto que los jóvenes, aunque cuentan con los conocimientos de prevención, no los considera necesarios en sus encuentros sexuales, tal vez debido a las presiones de la sociedad juvenil, e incrementan el riesgo de contagiarse del virus.

En opinión de Hidalgo ${ }^{(42)}$, el escaso interés de los universitarios hacia fuentes de información confiable como son los centros de salud influye de manera negativa en la permanencia de la infección, con implicaciones sociales, culturales y económicas. Según datos del Consejo Nacional de Población ${ }^{(41)}$ se ha demostrado que el acceso a la información acerca de las medidas preventivas en conjunto con revisiones médicas periódicas favorece una conducta saludable en los jóvenes. Asimismo, Sam y col ${ }^{(12)}$ opinan que es necesario abordar la importancia del sexo seguro y no solo cuando existan lesiones visibles en el área genital, sino como un método preventivo hacia la diseminación del VPH, por lo cual los jóvenes deben asistir a consultas ginecológicas y urológicas, lo cual reflejará en un futuro el resultado de las acciones en beneficio en la salud de la comunidad.

Las universitarias comentaron no haberse realizado la prueba de Papanicolaou $(79,9 \%)$ y, dado su inicio temprano a la vida sexual, es recomendable -contrario a la norma oficial mexicana (24) - promover estudios citológicos en jóvenes menores de 25 años de edad, dado que uno de los principales problemas del VPH es que la mayoría de los portadores desconoce su existencia ${ }^{(43)}$. Así, es de suma importancia diagnosticar y tratar las displasias desde el inicio de la afectación; los casos surgen a edades muy tempranas, constituyendo un grave problema de salud pública ${ }^{(44)}$.

En este sentido, las medidas de promoción a la salud deben de estar relacionadas con la madurez de la población. En este estudio, la edad se relacionó con el semestre que cursaban los alumnos como una representación de los universitarios de acuerdo a la madurez física, emocional y a la disponibilidad y acercamiento de información sobre el VPH. Sin embargo, los resultados sobre conocimiento y prácticas de prevención no reflejaron gran diferencia, lo que lleva a suponer que debe de orientarse políticas educativas que fomenten no solamente el conocimiento sobre esta patología sino al mismo tiempo fortalezca una salud reproductiva adecuada en los jóvenes.

Es importante reflexionar sobre la importancia de identificar el conocimiento y las prácticas de prevención sobre el VPH para tener una visión amplia del proceso contagioso del virus, de manera que en un futuro se cree estrategias que ayuden a los jóvenes en la adquisición de estilos de vida saludables y/o actitudes preventivas que limiten la diseminación de diversas infecciones. 
El presente estudio reveló que la mayor parte de los estudiantes desconocen con certeza la clínica, manejo y tratamiento de la infección y como consecuencia se mantienen inadvertidos ante la importancia del contagio. Los alumnos del área de la salud expresaron mayor comprensión referente al diagnóstico. Por otro lado, la población masculina expresó una edad menor en comparación con las mujeres en el inicio de la vida sexual, que fue reflejada en el mayor número de parejas. Esto cobra trascendencia si se considera que casi la mitad de los participantes no utilizaba el preservativo durante sus relaciones sexuales ni mostró interés de buscar información respecto al virus. Asimismo, la mayoría de las universitarias comentó no haberse realizado la prueba de Papanicolaou y la edad de los encuestados no influyó en el conocimiento ni prácticas de prevención contra el VPH.

A partir de dicha información, en primera instancia se concientizará a la comunidad universitaria sobre las repercusiones que conllevan la infección del VPH; asimismo, la divulgación de temas centrales como mecanismos de transmisión, métodos de prevención y características clínicas, con el objeto de otorgar mayor importancia a la educación sexual de los jóvenes y de esta forma prevenir complicaciones graves que pongan en peligro su vida, en especial si se considera que las actitudes de este grupo de edad son fácilmente modificables con la adquisición de conocimientos positivos, al ser potenciales perceptores y emisores, a su vez, de los conocimientos adquiridos.

\section{REFERENCIAS BIBLIOGRÁFICAS}

1. World Heatlh Organization. WHO. Guidance note: comprehensive cervical cancer prevention and control: a healthier future for girls and women. 2013. Disponible en: http://apps.who.int/iris/bitstre am/10665/85344/1/9789275317471_spa.pdf?ua=1

2. Castellsagué $X$. Natural history and epidemiology of HPV infection and cervical cancer. Gynecologic Oncology. 2008;110:s4-s7. doi:10.1016/j. ygyno.2008.07.045.

3. López A, Lizano M. Cáncer cervicouterino y el virus del papiloma humano: La historia que no termina. Cancerología. 2006;1:31-55.
4. Jiménez C, Correnti M, Salma N, Cavazza M, Perrone M. Detección del virus papiloma humano en entidades clinicas benignas de la cavidad bucal, mediante la reacción en cadena de la Polimerasa e hibridación molecular. Acta Odontológica Venezolana. 2000;39(2):10-5

5. Garcia P. Qué hay en el horizonte sobre el virus del papiloma humano, vacunas y el control del cáncer cervical. Rev peru med experim salud publica. 2007;24:272-9.

6. Cox T. Guia Provisional sobre el Uso del Test de VPH en combinación con la citología en el cribado cervical primario. En HPV Today, 2005;1:6.

7. Rivera R, Aguilera J, Larraín A. Epidemiología del virus papiloma humano (VPH). Rev Chil Obstet Ginecol. 2002;67(6):501-6. doi.org/10.4067/S071775262002000600013

8. Fernández CC, Delgado UJ. La carga del cáncer cervico-uterino y de la infección por virus del papiloma humano en México y en el mundo. Ginecol Obstet México. 2011;79(12):788-93.

9. Tami T. The new human papillomavirus (HPV) vaccina: pros and cons for pediatric and adolescents. Pediatric Nursing. 2008;34:429-31.

10. Tirado L, Mohar A, López M, García A, Franco F Borgues $G$. Factores de riesgo de cáncer cervicouterino, invasor en mujeres mexicanas. Salud Públ México. 2005;47:342-50.

11. Gómez GE, Gómez MS, Conde SE, Maganto PS, Navio NA. Allona A. Infección por papiloma virus en el hombre. Actas Urol Españolas. 2005;29(4):365-72.

12. Sam SS, Ortiz de la Peña CA, Lira PJ. Virus del papiloma humano y adolescencia. Ginecol Obstet México. 2011;79(4):214-24.

13. Ortunio CM, Guevara RH, Herrera LE. Conocimiento sobre el virus del papiloma humano en estudiantes de enfermería. Rev ginecol obstet. 2009;69(3):179-185.

14. México. Instituto Nacional de Estadistica y Geografía. INEGI. Estadísticas a propósito del dia mundial contra el cáncer datos nacionales. 2011. Disponible en: http://www.inegi.org.mx/inegi/contenidos/ espanol/prensa/contenidos/estadisticas/2011/ cancer11. asp?c $=2781$ \&ep $=51$

15. Sistema de universidades estatales de Oaxaca SUNEO. 2010 Estadisticas Universitarias. Disponible en: http://www.unsis.edu.mx/estadisticas/ SUNEO_Agosto_2010.pdf.

16. Arias Gómez ML, Pineda Lince SA. Conocimientos que tienen los estudiantes de una universidad pública de Manizales sobre el papilomavirus humano. Rev Hacia Promoción de la Salud. 2011;16(1):110-23.

17. Hernández CV, Aguilar CF, Toraño ZB, Sandoval $\mathrm{JL}$, Ceballos MZ. Identificación de mecanismos de transmisión del virus de papiloma humano en mujeres infectadas. Rev Enfermería IMSS. 2006;14(2):75-9.

18. Beatty BG, O'Connell M, Ashikaga T, Cooper K. Human papillomavirus (HPV) education in middle and high schools of Vermont. J Sch Health. 2003 Sep;73(7):253-7. doi: 10.1111/j.1746-1561.2003. tb06573.x

19. México. Instituto Nacional de Estadísticas y Geografia. INEGI. Mujeres y hombres en México 2013. Disponible en: http://cedoc.inmujeres.gob. $\mathrm{mx} /$ documentos_download/101234.pdf

20. México. Secretaria de Salud. SSA. Búsqueda intencionada de muertes maternas en México. 2008. Disponible en: http://www.cemece.salud.gob.mx/ descargas/pdf/BIMM_Informe2008.pdf

21. Organización Panamericana de la Salud. OPS.
Salud en las Américas: México. 2012. Disponible en: http://www.paho.org/saludenlasamericas/ index.php?option=com_content\&view=article\&id $=71$ \&ltemid $=67 \&$ lang $=e s$

22. 22. Garcia M, Mayoral M, Pintor R, Malvaez N, Méndez M. Perfil epidemiológico de la mortalidad por cáncer cervicouterino en Oaxaca 2000-2010. Rev evidencia e investigación clín. 2011;4(1):10-4.

23. Mejía A, Salas W. Costo-efectividad de la detección del virus del papiloma humano en los programas de tamización de cáncer de cuello uterino. Grupo de Economía de la Salud. Revista Médica. 2008;28:160-72.

24. México. Norma Oficial Mexicana NOM-014SSA2-1994, Para la prevención, detección, diagnóstico, tratamiento, control y vigilancia epidemiológica del cáncer cérvico uterino. 2007. Disponible en: http://www.salud.gob.mx/unidades/ cdi/nom/m014ssa294.pdf.

25. México. Norma Oficial Mexicana NOM-036SSA2-2002, Prevención y control de enfermedades. Aplicación de vacunas, toxoides, sueros, antitoxinas e inmunoglobulinas en el humano. 2003. Disponible en: http://www.salud.gob.mx/ unidades/cdi/nom/compi/acl036ssa202.html

26. Hernández-Carreño L, Padilla-Loredo S, QuinteroSoto ML. Factores de riesgo en adolescentes para contraer el virus del papiloma humano. Rev Digital Universitaria. 2012;13(9):3-16.

27. Murillo Z, Suárez Y, Hinestroza L, Bedoya AM, Sánchez IG, Baena A. Conocimiento de los estudiantes y docentes de la Escuela de Microbiología de la de la Universidad de Antioquia sobre la infección por el virus del papiloma humano. Rev Fac Nac Salud Pública. 2010;28(2):125-31.

28. Tamayo T, Varona S. Infección por papiloma virus humano en adolescente. Rev Cubana Obstet Ginecol. 2006;32:1-6.

29. Sánchez M, Uribe F, Conde C. La infección por el virus del papiloma humano, un posible marcador biológico de comportamiento sexual en estudiantes universitarios. Salud Pública México. 2002;44:442-7.

30. Mc Partland TS, Weaver BA, Lee SK, Koutsky L. Men's perceptions and knowledge of human papillomavirus (HPV) infection and cervical cancer. J Am Coll Health. 2005;53:225-30.

31. Dell D, Chen H, Ahmad F, Stewart D. Knowledge about human papillomavirus among adolescents. Obstet Gynecol. 2000;96:653-6.

32. Fox PA. Human papillomavirus and anal intraepithelial neoplasia. Current Op Inf Dis. 2006;1 9:62-6.

33. Wiesner C, Piñeros M, Trujillo LM, Cortés C, Ardila J. Human papillomavirus (HPV) vaccine acceptability amongst parents of adolescents in four Colombian areas. Rev salud pública. 2010;12(6):961-73. doi.org/10.1590/S0124-00642010000600008.

34. Herrero R, Castle PE, Schiffman M, Bratti MC, Hildesheim A. Epidemiologic profile of type-specific human papillomavirus infection and cervical neoplasia in Guanacaste, Costa Rica. J Infect Dis. 2005 Jun 1;191(11):1796-807.

35. Bermedo-Carrasco S, Xing Feng C, Peña-Sánchez JN, Lepnurm R. Predictors of having heard about human papillomavirus vaccination: Critical aspects for cervical cancer prevention among Colombian women. Gac Sanit. 2015;29:112-7. doi: org./10.1016/j.gaceta.2014.09.005

36. Kim JJ, Goldie SJ. Cost effectiveness analysis of including boys in a human papillomavirus vaccination programme in the United States. Brit Med J. 2009;339:b3884. doi: http://dx.doi.org/10.1136/ bmj.b3884. 
37. Falcao JJ, Vieira FL, Moura LE, De Oliveira RS Bezerra PA, Barbosa XL. Conocimientos de los estudiantes del área de salud sobre anticoncepción y prevención de enfermedades de transmisión sexual. Enfermeria global. 2009;8(15):1-12.

38. Agüero LB, Castañeda AL, Hernández GA, Durán DA. Virus del papiloma humano lo que saben los estudiantes masculinos de licenciatura. Rev Med IMSS. 2004;42(4):321-5.

39. Picón Ruiz MM. Conocimientos, percepciones y prácticas de mujeres jóvenes acerca del papiloma virus humano. ISP Collection. Paper 732. 2009. Disponible en:http://digitalcollections.sit.edu/ isp_collection/732.

40. Suárez-Allen RE, Puerto-Solís M, González-Losa MR. Interés en el conocimiento de las infecciones con virus del papiloma humano y el cáncer cervico- uterino entre estudiantes universitarios en Mérida, Yucatán, México. Rev Biomed. 2012;23:47-52.

41. México. Consejo Nacional de Población CONAPO. Situación actual de los jóvenes en México. Dirección de estudios sociodemográficos. 2012. Disponible en: http://www.conapo.gob.mx/publicaciones/ juventud/sdm/sdm2010/04.pdf.

42. Hidalgo MA. El cáncer cérvico-uterino, su impacto en México y por qué no funciona el Programa Nacional de Detección Oportuna. Rev Biomed. 2006;17(1):81-4.

43. Varela J. Casos de transmisión sexual, su aumento por causa de su desconocimiento por parte de los portadores. Diario Digital de Menorca, 2006:601.

44. Stoler ME. Prevención secundaria (detección) del cáncer cervicouterino. Ginecol Obstet México. 2011;79(12):808-15
Tipo de colaboración: Artículo original.

Los autores declaran no conflicto de intereses.

Responsable del trabajo:

Dr. Abisai Martínez-Sánchez

Guillermo Rojas Mijangos S/N Esq. Av. Universidad Col. Ciudad Universitaria, Miahuatlán de Porfirio Díaz,

Oaxaca. C.P 70800.

Teléfono: + 9512120407

Correo electrónico: abisai01@hotmail.com 